\title{
Nurses' compliance to patient and environmental safety guidelines in critical care units
}

\author{
Eman Aly Abd Elhamid ${ }^{1}$ *, Nehad Ezz Eldin Abdallah ${ }^{2}$, Safaa Mohamed Abd Elrahman ${ }^{3}$ \\ ${ }^{1}$ Clinical instructor Faculty of Nursing-Minia University, Egypt \\ 2 Professor of Nursing Administration, Faculty of Nursing, Cairo University, Egypt \\ ${ }^{3}$ Professor of Nursing Administration, Faculty of nursing - Minia University, Egypt \\ *Corresponding author E-mail: eman.aly100@yahoo.com
}

\begin{abstract}
The ability to provide safe and high-quality care to patients is the primary goal and the focus for many health care providers. Guidelines can be used to reduce adverse event in practice and to promote the delivery of high quality and evidence-based health care. The aim of the study to investigate nurses' compliance to patient and environmental safety guidelines in critical care units. Descriptive research design was utilized to achieve the aim of this study. The study was carried out at three of Minia University Hospitals in Minia city. All available nurses $(n=88)$ who were working in critical care units at time of data collection in the selected hospitals. An environmental and patient safety guidelines questionnaire was used to achieve the aim of this study. Results: less than two third of studied subject were not compliance to patient safety nurses', while nurses complied to environmental safety with $62.5 \%$ in critical care units; also, there is highly statistical significance between departments and nurses' compliance to patient safety. Thus, it was concluded that less than two third of studied subject were not compliance to patient safety. As well as environmental safety in critical care units was appropriate with $62.5 \%$ in critical care units. Recommendations: Staff development programs for nurses working in critical care units related to patient and environmental safety as well as ensuring that the organization's annual budget includes adequate resources to implement and evaluate health and safety activities.
\end{abstract}

Keywords: Compliance; Guidelines; Environment; Nurse; Safety.

\section{Introduction}

Patient safety is a relatively new concept in health care organization. Patient safety has been defined as the freedom from accidental or preventable injuries produced by medical care (Agency for Healthcare Research and Quality, 2005). The focus on patient safety began with the release of the Institute of Medicine (IOM) report "To Err Is Human" in 1999. The report estimated that between 44,000 and 98,000 patients die annually from medical errors. Errors in the care process can result in harm or injury that sometimes can leads to death (Shojania, et al., 2001). Adverse events can occur in the most efficient and safety conscious areas. One challenge facing critical care units today is the need for safety guidelines because the patient will be monitored closely for a critical medical condition with safety (Hassan, \& Ahmed, 2012)

Nurses represent the largest component of the healthcare work force; they are in a key position to improve patient safety through exerting significant influence in the health care system. So, it is important to understand nurses' compliance to patient safety and to comprehend what factors influence their views. This is based on the number of nurses as the largest segment in the hospital. In carrying out its functions, a nurse is a staff who has the most contact with patients (24 hours continuously) (Powell-Cope, Nelson, \& Patterson 2008).

Nurses should implement patient safety and concern when providing nursing care to the patients. Nurses must also involve cognitive, affective, and action that promotes patient safety. Perception of the nurses to maintain the safety of the patient is important in the prevention and control of patient safety. The extent of the nurses' role allows the risk of errors and service (Amiri, Khademian\& Nikandish, 2018).

The Critical Care Unit is a specialized area of a hospital that provides comprehensive and continuous care for patients who are critically ill. Maintaining patient safety is becoming a crucial objective for nurses in critical care units. Critical care patients are more prone to experience medical errors because they are seriously ill and require continuous supervision. Their care is more complex than that required by other hospital patients because of the advanced medical technology, coordination between the multiple healthcare providers, and administration of many medications required (Gutsche \& Kohl, 2007).

Compliance has been defined as the extent to which certain behavior is in accordance with the physicians' instructions or health care advice. The recent studies have linked global measures of a safety culture to nurses and other health team compliance with safe work practices; also the exposure to incidents, regardless of the outcome, may be extremely burdensome to health team as well as to organizations (Sexton, et al., 2011). Compliance can be influenced or controlled by a variety of factors like culture, economic and social factors, 
self-efficacy, and lack of knowledge or means. Guidelines are a group of steps and procedures that guide an individual's behavior exist in a variety of settings (including health care settings), but people do not always comply with them (Efstathiou, et al. 2011).

The aim of guidelines is to provide a comprehensive range of evidence-based recommendations for interventions to be applied for patient care during the periods of hospitalization, and for the prevention of hospital hazards or risk for patient during hospitalization. Therefore, patient safety is a fundamental principle of health care which should have guidelines. A number of high-income countries have published studies illustrating that significant numbers of patients are harmed during health care, either resulting in permanent injury, increased length of stay in health care facilities, or even death (WHO, 2017; WHO, 2018).

\section{Significance of the study}

During the clinical training of the nursing students at Minia University Hospitals, It has been observed at the studied hospitals that nurses' staff had various problems related to environment and patient safety in critical care units such as patient bed sores, patient falling from bed, no infection control, improper waste management, and medication errors.

In critical care units, the critical ill patients had more complexity of care which done in a highly technologically advanced environment, and nurse's compliance to patient safety are rarely formally evaluated or considered during the design or updating of safety programs. Despite, a strongly nurse's compliance to patients' safety contributes to a positive patients safety culture that necessary to maintain patient safety and avoid patient harm. In addition, starting point for improving quality nursing care is to investigate nurse's compliance to patient's and environment safety.

\section{Aim of the study}

The aim of this study was to investigate Nurses' compliance to patient and environmental safety guidelines in critical care units.

\section{Research questions}

- What is the level of nurses' compliance to patient and environmental safety guidelines in critical care units?

- What is relation between patient safety and nurses' personal data?

\section{Subjects and methods}

\subsection{Research design}

Descriptive design was utilized in this study.

\subsection{Setting}

The study was carried out at critical care units of three University Hospitals: Minia; Obstetric and Pediatric, and Cardiothoracic including eight units as follows:

- Minia University Hospital: (Medical intensive care unit, Neurosurgery Intensive care unit, Intensive care unit).

- Obstetric and Pediatric University Hospital: (Obstetrics intensive care unit, Pediatric intensive care unit).

- Cardiothoracic University Hospital: (Coronary care unit, Cardiothoracic intensive care unit, Chest intensive care unit.

\subsection{Subjects}

A convenient sample of available nurses $(n=88)$ who were working in critical care units during reliability phases of proposed tool except nurses who having less than one year of experience in critical care units.

\subsection{Tool of data collection}

Patient and environmental safety guidelines Observational checklist, this tool was developed by authors (Abd Elhamid ${ }^{1}$; Abdallah ${ }^{2} ;$ Abd Elrahman $^{3}$ ) based on extensive review literature such as: WHO (2011), Hirumalai (2010), Ali (2010), Sexton et al., (2006), and it included three parts as follows:

- 1st part was including: personal characteristics data sheet for nurses: (age, gender, years of experience, department, hospital name, and qualification).

- 2nd part: this part was concerning patient safety guidelines; and it consisted of 47 items categorized in 4 subscales namely; administrating medication ( 24 items), bedside nurse ( 6 items), bedsores ( 3 items) and patient falling (14 items). The responses were rated on 2 point scale: (done $=1$ and not done $=0$ ). The scoring system of this part was classified as follows: not compliance to patient safety guidelines $(<75 \%)$, and compliance to patient safety guidelines $(\geq 75 \%)$.

- $\quad 3 r d$ part: this part was concerning environmental safety; and it consisted of 97 items categorized in 13 subscales namely; work environment (10 items); infection control (15 items); personal protective equipment ( 8 items); ventilation (4 items); water (4 items); Furniture and equipment safety ( 8 items); Handling and storage of materials (4 items); Maintenance (3 items); Doors and exits (4 items); Fire (prevention and protection) (11 items); Lighting and electricity safety (12 items); Waste disposal (10 items) and Housekeeping ( 4 items). The responses were rated on 2 point scale: (compliance $=1$ and not compliance $=0$ ). The scoring system of this part was classified as follows: not compliance to environmental safety $(<75 \%)$, and compliance to environmental safety $(\geq$ $75 \%$.)

\subsection{Validity and reliability}


The tool was revised and validated by one thirty experts in the field of study to assess its validity. And for the reliability of the tool, it was done using alpha coefficient to measure the internal Consistency reliability and was (0.991).

\subsection{Pilot study}

A pilot study was conducted on 8 head nurse (10\%) that were included in the study subjects; they selected randomly from the different critical care units in selected hospitals in order to; check and ensure clarity and applicability of the tools; and to identify obstacles and problems that may be encountered during data collection. No changes occurred in the tool and it was put in its final form.

\subsection{Procedure}

- An official permission was obtained from Dean of Faculty of Nursing- Minia University.

- An official permission was obtained from directors of hospitals

- An extensive review of the related literature which covering various aspects of patient and environment safety was done. The observational checklist was developed in sixth months from beginning of August 2016 to end of January 2017.

- The researcher was collecting data in two months from beginning of April 2019 to end of May 2019 by used environmental and patient safety guidelines.

\subsection{Ethical consideration}

- This study was granted approval by the Ethical Committee of the Faculty of Nursing, Minia University.

- The aim of the study was explained to nurses participate in the study.

- Oral informed consents were obtained from all the participants before the data collection.

- The study participants were reassured that their participation was voluntary and they had the right to withdraw from the study at any time if they want that.

- The study participants were reassured that their anonymity was maintained although the study and collected information wouldn't be used except for the purpose of this study and written consent were obtained.

\subsection{Statistical analysis}

Data were analyzed using the statistical package for social science (SPSS) version 20. Numerical data were expressed as mean and SD. Quantitative data were expressed as frequency and percentage. For quantitative data, comparison between two variables was done using $\mathrm{t}$-test, and comparison between more than two variables used ANOVA test. Relations between different numerical variables were tested using Pearson correlation. Probability (p-value) less than 0.05 was considered significant and less than 0.001 was considered highly significant.

\section{Results}

Table (1) shows that majority of nurses (87.5\%) who worked in critical care unit aged from (20-30) years; more than two third of nurses $(69.3 \%)$ were female, and more than one third of nurses (38.6\%) worked in Minia University Hospital. Concerning the department, it was noted that highest percent $(26.1 \%)$ of nurses worked in coronary care unit; less than three quarter of them $(70.5 \%)$ had less than five years of experience; and more than half of them (58\%) graduated from clinical institute.

Figure (1) shows that about near to two third (64.8) of nurses who worked in critical care units had not compliance to patient safety.

Figure (2) shows that environmental safety in critical care units was appropriate with $62.5 \%$.

Table (2) displays that the highest percent of nurses not complied with patient safety in critical care unit regarding all patient safety dimensions: bedside nurses, patient falling guidelines, administrating medication, and bedside nurses (67\%, 61.4\%, 60.2\%, and 58\% respectively).

Table (3) reveals that personal protective equipment; water safety; ventilation; handling and storage of materials, maintenance and housekeeper were appropriate with $75 \%$ in critical care units. While, the fire (prevention and protection), infection control, as well as furniture and equipment safety were not appropriate $(75 \%, 62.5 \%$ and $62.5 \%$ respectively).

Table (4) shows that there is highly statistical significance between age, hospital and departments as regard to nurses' compliance to patient safety ( $\mathrm{p}=0.003,0.001$, and 0.001 respectively).

Table (5) shows that there is highly statistical significance department regarding the nurses compliance to patient safety in all dimensions $(p=0.001)$. The highest total mean score were for the nurses who worked in Chest intensive care unit and Neurosurgery Intensive care unit (45.0+0.0), followed by Medical intensive care unit (43.1+3.5), then Cardiothoracic intensive care unit (40.5+7.6)

Table 1: Percentage Distribution of the Study Sample According to Their Personal Data

\begin{tabular}{|c|c|c|}
\hline Personal data & $\begin{array}{l}\text { Nurs } \\
\text { No }\end{array}$ & $\%$ \\
\hline \multicolumn{3}{|l|}{ Age } \\
\hline$<30$ & 77 & 87.5 \\
\hline $31-40$ & 8 & 9.1 \\
\hline$>40$ & 3 & 3.4 \\
\hline Mean \pm SD & \multicolumn{2}{|c|}{$24.4 \pm 6.5$ years } \\
\hline \multicolumn{3}{|l|}{ Sex } \\
\hline Male & 27 & 30.7 \\
\hline Female & 61 & 69.3 \\
\hline \multicolumn{3}{|l|}{ Hospital } \\
\hline Minia University Hospital & 34 & 38.6 \\
\hline Obstetric \& Pediatric University Hospital & 17 & 19.3 \\
\hline Cardiothoracic University Hospital & 37 & 42.0 \\
\hline
\end{tabular}


Department

Medical intensive care unit

Coronary care unit

Neurosurgery Intensive care unit

Intensive care unit.

Obstetrics intensive care unit.

Pediatric intensive care unit.

Cardiothoracic intensive care unit.

Chest intensive care unit.

Years of experience

$<5$

6-10

11-15

16-20

21-25

Mean \pm SD

Qualification

Diploma nurse

Clinical institute

Bachelor of nursing

\begin{tabular}{ll}
11 & 12.5 \\
23 & 26.1 \\
9 & 10.2 \\
14 & 15.9 \\
9 & 10.2 \\
8 & 9.1 \\
7 & 8.0 \\
7 & 8.0 \\
62 & \\
16 & 70.5 \\
4 & 18.2 \\
2 & 4.5 \\
4 & 2.3 \\
$5.2 \pm 5.9$ years & 4.5 \\
& \\
15 & 17.0 \\
58 & 65.9 \\
15 & 17.0 \\
\hline
\end{tabular}

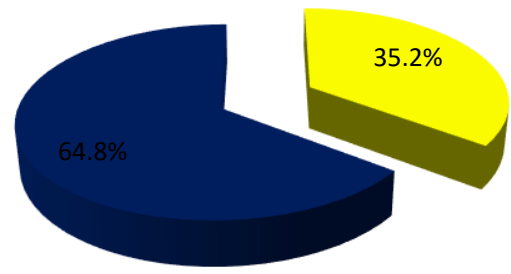

compliance

not compliance

Fig. 1: Distribution of Total Score About Nurses' Compliance Toward Patients' Safety (N=88)

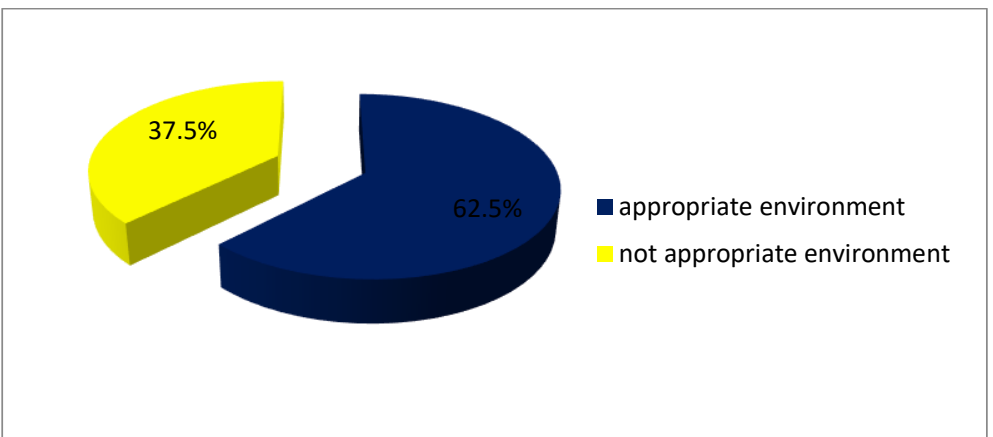

Fig. 2: Distribution of Total Score About Environmental Safety in Critical Care Units (N=8 Units).

Table 2: Frequency Distribution of Nurses' Compliance Toward Dimensions of Patients' and Environment Safety (N=88)

\begin{tabular}{|c|c|c|c|c|c|}
\hline \multirow{3}{*}{\multicolumn{2}{|c|}{ Dimensions of patient safety }} & \multicolumn{4}{|c|}{ Nurses $(n=88)$} \\
\hline & & \multicolumn{2}{|c|}{ Compliance } & \multicolumn{2}{|c|}{ Not Compliance } \\
\hline & & No & $\%$ & No & $\%$ \\
\hline 1. & Administering medication & 35 & 39.8 & 53 & 60.2 \\
\hline 2. & Bedside nurses & 37 & 42 & 51 & 58 \\
\hline 3. & Bedsores prevention & 29 & 33 & 59 & 67 \\
\hline 4. & Patient falling prevention & 34 & 38.6 & 54 & 61.4 \\
\hline
\end{tabular}

Table 3: Percentage Distribution of Nurses' Compliance to Environmental Safety in Critical Care Units

\begin{tabular}{|c|c|c|c|c|c|}
\hline \multirow{2}{*}{\multicolumn{2}{|c|}{ Dimension of environmental safety }} & \multicolumn{2}{|c|}{ Compliance } & \multicolumn{2}{|c|}{ Not compliance } \\
\hline & & \multirow{2}{*}{$\frac{\text { No }}{44}$} & \multirow{2}{*}{$\frac{\%}{50.0}$} & \multirow{2}{*}{$\frac{\text { No }}{44}$} & \multirow{2}{*}{$\frac{\%}{50.0}$} \\
\hline 1. & Work environment & & & & \\
\hline 2. & Infection control & 33 & 37.5 & 55 & 62.5 \\
\hline 3. & Personal protective equipment & 66 & 75.0 & 22 & 25.0 \\
\hline 4. & Water safety & 66 & 75.0 & 22 & 25.0 \\
\hline 5. & Ventilation & 66 & 75.0 & 22 & 25.0 \\
\hline 6. & Furniture and equipment safety & 33 & 37.7 & 55 & 62.5 \\
\hline 7. & Handling and storage of materials & 66 & 75.0 & 22 & 25.0 \\
\hline 8. & Maintenance & 66 & 75.0 & 22 & 25.0 \\
\hline 9. & Doors and exits & 55 & 62.5 & 33 & 37.5 \\
\hline 10. & Fire (prevention and protection) & 22 & 25.0 & 66 & 75.0 \\
\hline 11. & Lighting and electricity safety & 55 & 62.5 & 33 & 37.5 \\
\hline 12. & Waste disposal & 44 & 50.0 & 44 & 50.0 \\
\hline 13. & Housekeeper & 66 & 75.0 & 22 & 25.0 \\
\hline
\end{tabular}

Table 4: Relation Between Nurses' Compliance Toward Patients' Safety and Their Personal Characteristics $(\mathrm{N}=88)$ 


\begin{tabular}{|c|c|c|c|c|c|}
\hline \multirow[t]{2}{*}{ Personal data } & \multicolumn{2}{|c|}{ Patient safety } & Con & nce & \multirow[t]{2}{*}{ Fisher exact $\mathrm{P}$ value } \\
\hline & No & $\%$ & No & $\%$ & \\
\hline Age & 53 & 602 & 24 & 273 & \multirow{4}{*}{$\begin{array}{l}11.76 \\
0.003\end{array}$} \\
\hline$<30$ & 53 & 60.2 & 24 & 27.3 & \\
\hline $31-40$ & 1 & 1.1 & 7 & 8.0 & \\
\hline$>40$ & & & & & \\
\hline \multicolumn{6}{|l|}{ Sex } \\
\hline Male & 14 & 15.9 & 13 & 14.8 & 2.85 \\
\hline Female & 43 & 48.9 & 18 & 20.5 & 0.09 \\
\hline Hospital & 15 & 170 & 19 & & \multirow{4}{*}{$\begin{array}{l}15.72 \\
0.001\end{array}$} \\
\hline Minia University Hospital & 15 & 17.0 & 0 & & \\
\hline Obstetric \& Paediatric University Hospital & 17 & 19.3 & 0 & $0.0 \%$ & \\
\hline Cardiothoracic University Hospital & 25 & 28.4 & 12 & 13.6 & \\
\hline \multicolumn{6}{|l|}{ Department } \\
\hline Intensive care unit & 14 & $15.9 \%$ & 0 & $0.0 \%$ & \multirow{8}{*}{$\begin{array}{l}77.75 \\
0.001\end{array}$} \\
\hline Medical intensive care unit & 1 & $1.1 \%$ & 10 & $11.4 \%$ & \\
\hline Chest intensive care unit & 0 & $0.0 \%$ & 7 & $8.0 \%$ & \\
\hline Obstetrics intensive care unit & 9 & $10.2 \%$ & 0 & $0.0 \%$ & \\
\hline Neurosurgery Intensive care unit & 0 & $0.0 \%$ & 9 & $10.2 \%$ & \\
\hline Paediatric intensive care unit. & 8 & $9.1 \%$ & 0 & $0.0 \%$ & \\
\hline Cardiothoracic intensive care unit. & 2 & $2.3 \%$ & 5 & $5.7 \%$ & \\
\hline Coronary care unit & 23 & $26.1 \%$ & 0 & $0.0 \%$ & \\
\hline Qualification & & & 5 & & \multirow{4}{*}{$\begin{array}{l}0.691 \\
0.708\end{array}$} \\
\hline Diploma nurse & $\begin{array}{l}10 \\
36\end{array}$ & $\begin{array}{l}11.4 \\
409\end{array}$ & $\begin{array}{l}5 \\
22\end{array}$ & 5.7 & \\
\hline Clinical institute & 36 & $\begin{array}{l}40.9 \\
125\end{array}$ & 22 & 25.0 & \\
\hline Bachelor of nursing & 11 & 12.5 & 4 & 4.5 & \\
\hline Years of experience & 39 & 44.3 & 23 & 26.1 & \multirow{6}{*}{$\begin{array}{l}1.860 \\
0.793\end{array}$} \\
\hline $0-5$ & 11 & 12.5 & 5 & 5.7 & \\
\hline $6-10$ & 2 & 2.3 & 2 & 2.3 & \\
\hline $11-15$ & 2 & 2.3 & 0 & $\begin{array}{l}2.3 \\
0.0\end{array}$ & \\
\hline $16-20$ & 2 & 3.5 & & 0.0 & \\
\hline $21-25$ & 3 & 3.4 & 1 & 1.1 & \\
\hline
\end{tabular}

Table 5: Comparison Between Different Departments Regarding Nurse's Compliance to Patient's Safety (N=88)

\begin{tabular}{|c|c|c|c|c|c|}
\hline $\begin{array}{l}\text { Dimensions } \\
\text { Department }\end{array}$ & Administering medication & Bede side nurses & Bed sores & Patient falling & Total \\
\hline \multirow{2}{*}{$\begin{array}{l}\text { Intensive care unit } \\
\text { Medical intensive care unit }\end{array}$} & $10.8+3.2$ & $3.00+0.0$ & $1.00+0.0$ & $9.00+0.0$ & $23.8+3.2$ \\
\hline & $23.0+0.0$ & $5.00+0.0$ & $2.09+1.04$ & $13.09+3.0$ & $43.1+3.5$ \\
\hline Chest intensive care unit & $23.0+0.0$ & $5.00+0.0$ & $3.00+0.0$ & $14.0+0.0$ & $45.0+0.0$ \\
\hline Obstetrics intensive care unit & $10.0+0.0$ & $2.77+.44$ & $1.00+0.0$ & $9.11+.78$ & $22.8+.78$ \\
\hline Neurosurgery Intensive care unit & $23.0+0.0$ & $5.00+0.0$ & $3.00+0.0$ & $14.0+0.0$ & $45.0+0.0$ \\
\hline Paediatric intensive care unit & $13.2+6.0$ & $3.12+1.55$ & $1.00+0.0$ & $5.50+2.07$ & $22.8+6.4$ \\
\hline Cardiothoracic intensive care unit & $18.5+7.6$ & $5.00+0.0$ & $3.00+0.0$ & $14.0+0.0$ & $40.5+7.6$ \\
\hline Coronary care unit & $10.0+0.0$ & $3.00+0.0$ & $1.00+0.0$ & $9.00+0.0$ & $23.0+0.0$ \\
\hline ANOVA & 44.32 & 53.05 & 70.04 & \multirow{2}{*}{59.610 .001} & 109.3 \\
\hline $\mathrm{P}$ value & 0.001 & 0.001 & 0.001 & & 0.001 \\
\hline
\end{tabular}

*ANOVA test was used for quantitative data * Significant difference in between groups ( $\mathrm{p}$ value $\leq 0.05$ ).

\section{Discussion}

Guidelines that guide an individual's behavior exist in a variety of settings (including health care settings), but people do not always comply with them. Compliance can be influenced or controlled by a variety of factors like culture, economic and social factors, selfefficacy, and lack of knowledge or means (Joint Commission, 2017). Moreover, patient safety is a constant domain in any framework for quality healthcare. All organizations should ensure the safety of their patients through the adoption of safe practices. Defining these practices and how to implement them through policies and procedures is the starting point. Policies and procedures foster a right culture that supports standardization and documentation that helps create consistency in patient safety practices thus minimizing patient harm (Efstathiou, et al. 2011).

The result of the current study majority of nurses who worked in critical care unit aged from (20-30) years; more than two third of nurses were female, and more than one third of nurses worked in Minia University Hospital. Concerning the department, it was noted that highest percent of nurses worked in coronary care unit; less than three quarter of them had less than five years of experience; and more than half of them graduated from clinical institute.

The result of the current study, showed that less than two third of studied subject were not compliance to patient safety. This may attribute to nursing work environment in the critical care unit which is described as stressful, wearing, fatiguing and overloading, especially regarding the working hours. Also, nurses should be prepared to deliver care to patients with important hemodynamic alterations at any time, which demand specific knowledge and great skill to make decisions and put them in practice in due time.

This result was consistent with Colón-Emeric et al., (2010) who stated that preventable errors in patient care continue to occur despite the efforts of regulators, government, and healthcare organizations due to workload especially in critical care units. These errors cause harm to patients, and present financial and operational burdens on an organization, and the number and impact of errors is astounding. Moreover, Julianto, Thiangc, and Boonyoung (2016) mentioned that the hospital have role important in order to enhance quality of care and availability the equipment's as well as decrease the impact of crisis healthcare such as harm for patient, cost for healthcare, anticipation of workload thereby nurses and other healthcare worker can improve performance of patient safety and work environment 
Moreover, Suliman (2018) added that nurses demonstrated low compliance with some safety precautions applied to ICU patients. For example, nurses were less compliant with some safety precautions such as changing the patient's position every two hours, and fixing an indwelling catheter to the bed.

Also, Bassuni and Bayoumi (2015) mentioned that the high nursing workload and fatigue have been identified as major negative contributors to patient safety; so providing safe and error-free care is the priority of all health care professionals. While, this result was inconsistent with Mazhari et al., (2014) who found that compliance rate of patient safety standards was about $72 \%$.

The finding of present study revealed that nurses' in critical care units complied to environmental safety with $62.5 \%$. This result might be attributed that critical care units is highly specified and sophisticated area in any hospital in which these units are specifically designed, staffed, located, furnished and equipped, dedicated to management of critically ill patients; therefore, the nurses can be more complied to environment safety due to availability of resources.

This finding was in accordance with Backes et al., (2015) who mentioned that the critical care units professionals showed great concern with regard to the environment, this due to the particularly size of the space where patients are found, the presence of natural light. In the critical care units there is a need to use many materials and items of equipment, and they need to be suitable and sufficient. Critical care units' environment involves suitable technology; that is to say technological appliances, such as infusion pumps, respirators, cardiac monitors, and others.

The result of the current study indicated that more than half of studied sample not complied with administrating medication safety. This result may be due to lack of nurses' knowledge and experience related to medication safety and heavy workload in critical care units. This finding was consistent with (Cheragi et al.,2013) who emphasized that medication errors are among the most common nursing errors, the nurse spends time and energy in the administration process carrying the responsibility for accurately administration of the right drug. For that, the nurse should encompass the knowledge about the reason forgiving a certain drug, its action, side effects, usual dose and recognize mistakes in prescriptions.

The result of the present study illustrated that the majority of nurses worked in critical care units not complied with bed sores prevention and patient fall prevention. This result was might be attributed that decrease of nurses number in critical care units can lead to increase workload which affect on quality of their work. This finding supported by (Warelow et al., 2008) who stated that increased workloads, time pressures, frequent work interruptions and the inability to predict what will happen in their work environments contribute quality of care.

Also, Canadian Nurse Association, (2004) reported that heavy workloads may prevent experienced nurses from guiding their less experienced colleagues. Nurses do not complete reports when errors happen because they do not have time. Therefore, the patient safety regarding medication administration, bedsores and preventing falls are major indicators to the quality of care that provided in the hospital, this was consistent with Twigg et al., (2012) who added that patient safety issues such as patient identification, falls, and medication safety has been considered as a major indicator of healthcare quality

The result of current study revealed that there is highly statistical significance between age, and nurses' compliance to patient safety. This result might be due to new graduated nurses have not sufficient knowledge and unable to incorporate theory to practice. This result was consistent with Ammouri et al., (2015) who reported that nurses who had more years of experience and who were working in teaching hospitals had more perception of patient safety.

The result of this study showed that there is highly statistical significance between departments and nurses' compliance to patient safety. The highest total mean score were for the nurses who worked in chest intensive care unit and neurosurgery Intensive care unit, followed by Medical intensive care unit, then cardiothoracic intensive care unit. While lowest total mean score were for the nurses who worked intensive care unit, obstetric Intensive care unit, Paediatric intensive care unit and coronary care unit, have the most critical cases, and patients required more complicated care than patients in other departments. This may be attributed to proper nurse/patient ratio, present of sufficient and work environment are more organized in critical care units than others units.

This was consistent with Kane-Gill et al., (2012) who stated that patients in intensive care are typically at a higher risk for poor clinical outcomes as compared to general care patients, as a result of their clinical conditions. Clinical condition has been shown to be a risk factor for adverse events. Therefore, seriously ill patients are more likely to experience an adverse event than other hospitalized patients

\section{Conclusion}

In the current study, it was noted that less than two third of studied subject were not compliance to patient safety. As well as environmental safety in critical care units was appropriate with $62.5 \%$ in critical care units.

\section{Recommendations}

Based on the findings of the current study it was recommended that

1) Staff development programs for nurses working in critical care units related to patient and environmental safety.

2) Develop a strategic plan for patient and environmental safety

3) Improve nurses reporting skills of incidents related to patient safety

4) Ensuring that the organization's annual budget includes adequate resources to implement and evaluate health and safety activities.

\section{References}

[1] Agency for Healthcare Research and Quality. (December 2005). National healthcare quality report. Rockville, MD: U.S. Department of Health and Human Services, Agency for Healthcare Research and Quality. AHRQ Pub. No. 06-0018. Available at: www.ahrq.gov/qual/nhqr05/nhqr05.htm. Retrieved on May 3, 2019.

[2] Ali. A. (2010). Assessment of nurses' awareness about availability and importance of applicability of safety measures, published master thesis, Assuit University, 92.

[3] Amiri, M., Khademian, Z., \& Nikandish, R. (2018). The effect of nurse empowerment educational program on patient safety culture: a randomized controlled trial. BMC medical education, 18(158):1-8. https://doi.org/10.1186/s12909-018-1255-6.

[4] Ammouri, A. A., Tailakh, A. K., Muliira, J. K., Geethakrishnan, R., \& Al Kindi, S. N. (2015). Patient safety culture among nurses. International Nursing Review, 62(1), 102-110. https://doi.org/10.1111/inr.12159. 
[5] Backes, M. T. S., Erdmann, A. L., \& Büscher, A. (2015). The living, dynamic and complex environment care in intensive care unit. Revista latinoamericana de enfermagem, 23(3), 411-418. https://doi.org/10.1590/0104-1169.0568.2570.

[6] Bassuni, E. M., \& Bayoumi, M. M. (2015). Improvement critical care patient safety: using nursing staff development strategies, at Saudi Arabia. Global journal of health science, 7(2), 335-343. https://doi.org/10.5539/gjhs.v7n2p335.

[7] Canadian Nurse Association. (2004). Nurse and patient safety: discussion paper Faculty of Nursing, Toronto university, Ottawa.

[8] Cheragi, M. A., Manoocheri, H., Mohammadnejad, E., \& Ehsani, S. R. (2013). Types and causes of medication errors from nurse's viewpoint. Iranian journal of nursing and midwifery research, 18(3), 228-231.

[9] Colón-Emeric, C. S., Plowman, D., Bailey, D., Corazzini, K., Utley-Smith, Q., Ammarell, N., Anderson, R. (2010). Regulation and mindful resident care in nursing homes. Qualitative Health Research, 20(9), 1283-1294. https://doi.org/10.1177/1049732310369337.

[10] Efstathiou, G., Papastavrou, E., Raftopoulos, V., Merkouris, A. (2011). Factors influencing nurses' compliance with Standard Precautions in order to avoid occupational exposure to microorganisms: A focus group study." BMC nursing : 10(1), 1-12. https://doi.org/10.1186/1472-6955-10-1.

[11] Gutsche, J. T., \& Kohl, B. A. (2007). Who should care for intensive care unit patients?. Critical care medicine, 35(2): S18-S23. https://doi.org/10.1097/01.CCM.0000252907.47050.FE.

[12] Hassan, M. R., \& Ahmed, T. S. (2012). Patient safety assessing nurses' compliance. Journal of American Science, 8(1), 748-755.

[13] Hirumalai, A. (2010). Nursing compliance with standard fall prevention protocol among acute care hospital nurses, Published master thesis, University of Nevada Las Vegas, 44.

[14] Joint Commission. (2017). The essential role of leadership in developing a safety culture. Published by the Department of Corporate Communications, Sentinel event alert (issue 57).

[15] Julianto R, Thiangc P, \& Boonyoung N. (2016). Review: Factors related to compliance of nurses toward the use of personal protective equipment in the hospital. Available at http://applications.emro.who.int/dsaf/EMROPUB_2016_EN_18948.pdf?ua=1.

[16] Kane-Gill, S., \& Weber, R. J. (2006). Principles and practices of medication safety in the ICU. Critical care clinics, 22(2): 273-290. https://doi.org/10.1016/j.ccc.2006.02.005.

[17] Mazhari, Z., Hesam, S., \& Zarnaq, R. K. (2014). The compliance rate of patient safety standards in hospital: a compare and analysis at Tehran hospitals. Journal of American Science, 4(14), 12-17.

[18] Powell-Cope G, Nelson AL, Patterson ES. (2008). Patient Care Technology and Safety. In: Hughes RG, editor. Patient Safety and Quality: An Evidence-Based Handbook for Nurses. Rockville (MD): Agency for Healthcare Research and Quality (US); Apr. Chapter 50.

[19] Sexton, J. B., Helmreich, R.L., Neilands, T.B., Rowan, K., Vella, K., Boyden, J., Thomas, E.J. (2006). The safety attitude questionnaire psychometric properties, benchmarking data and emerging research. BMC, Health services research, 6(44), 1-10. https://doi.org/10.1186/1472-6963-6-44.

[20] Sexton, J.B., Berenholtz, S.M, Goeschel CA, Watson, S.R., Holzmueller, C.G., Thompson, D.A., Hyzy, R.C., Marsteller, J.A., Schumacher, K., \& Pronovost, P.J. (2011). Assessing and improving safety climate in a large cohort of intensive care units. Crit Care Med; 39(5):934-939. https://doi.org/10.1097/CCM.0b013e318206d26c.

[21] Shojania, K.,G., Duncan, B.W., McDonald, K.M., Wachter, R.M., Markowitz, A.J. (2001). Making health care safer: a critical analysis of patient safety practices." Evid Rep Technol Assess (Summ), 43(1): 1-668.

[22] Suliman M. (2018). Measuring Nurses' Compliance with Safety Precautions for Patients in Intensive Care Units. International Journal of Nursing Education, 10(4), 84-89. https://doi.org/10.5958/0974-9357.2018.00108.3.

[23] Twigg, E., Christine, D., \& Gemma, E. (2013). The critical role of nurses to the successful implementation of the National Safety and Quality Health Service Standards." Australian Health Review 37.4 541-546 https://doi.org/10.1071/AH12013.

[24] Warelow, P., Edward, K. L., \& Vinek, J. (2008). Care: What nurses say and what nurses do. Holistic Nursing Practice, 22(3):146-153. https://doi.org/10.1097/01.HNP.0000318023.53151.33.

[25] World Health Organization (WHO). Global guidelines for the prevention of surgical site infection. 2018. Available at https://www.who.int/publications/guidelines/patient_safety/en/ retrieved on June, 1, 2019.

[26] World Health Organization (WHO). Patient safety: making health care safer. (2017). Available at https://apps.who.int/iris/bitstream/handle/10665/255507/WHO-HIS-SDS-2017.11-eng.pdf retrieved on June, 1, 2019.

[27] World Health Organization. Patient safety assessment manual. (2011). Available at http://applications.emro.who.int/dsaf/emropub_2011_1243.pdf. Retrieved on June, 10, 2019. 